\title{
BreastLight Apparatus Performance in Detection of Breast Masses Depends on Mass Size
}

\author{
Seyed Mostafa Shiryazdi ${ }^{1}$, Saeed Kargar $^{1 *}$, Hossein Taheri-Nasaj ${ }^{3}$, Hossein \\ Neamatzadeh $^{4}$
}

\begin{abstract}
Background: Accurate measurement of breast mass size is fundamental for treatment planning. We evaluated performance of BreastLight apparatus in detection breast of masses with this in mind. Materials and Methods: From July 2011 to September 2013, a total of 500 women referred to mammography unit in Yazd, Iran for screening were recruited to this study. Performance of BreastLight in detection breast masses regard their sizeing, measured with clinical breast examination (CBE), mammography and sonography, was assessed. Sonographic and mammography examinations were performed according to breast density among women in two groups of women younger $(n=105)$ and older $(n=395)$ than 30 years. Size correlations were performed using Spearman rho analysis. Differences between mass size as assessed with the different methods (mammography, sonography, and clinical examination) and the BreastLight detection were analyzed using $\mathbf{X}^{2}$-trend test. Results: Performance of the BreastLight in detection of lesions smaller than or equal to $1 \mathrm{~cm}$ assessed by CBE, mammography and sonography was $4.4 \%, 7.7 \%$ and $12.5 \%$ and for masses larger than $4 \mathrm{~cm}$ was $65 \%, 100 \%$ and $57.1 \%$, respectively. The performance of BreastLight in detection was significantly increased with larger masses $(\mathbf{p}<\mathbf{0 . 0 0 1})$. Conclusions: We conclude that clinical measurement of breast cancer size is as accurate as that from mammography or ultrasound. Accuracy can be improved by the use of a simple formula of both clinical and mammographic measurements.
\end{abstract}

Keywords: Breast masses - BreastLight - mammography - sonography - clinical breast examination

Asian Pac J Cancer Prev, 16 (3), 1181-1184

\section{Introduction}

Breast cancer is one of the major health complications among women worldwide (Fouladi et al., 2013). The American Cancer Society's estimates indicate approximately 1.3 million new cases of invasive breast cancer were diagnosed globally in 2007; and nearly 500,000 women died from the disease.

Breast cancer size is one of the main prognostic indicators and a determining factor for surgical treatment planning (Nguansangiam et al., 2009; Luparia et al., 2013). The ability to accurately and reliably measure the size of the breast cancer prior to any surgical treatment or primary medical therapy is essential. With the development of minimally invasive breast surgical techniques and the increasing adoption of neoadjuvant therapy, the ability to correctly determine maximum tumor dimension noninvasively is becoming more important (Hieken et al., 2001; Azizun-Nisa et al., 2008; Tham et al., 2009). The decision to offer patients primary medical therapy for operable breast cancer depends principally on tumour size at presentation, with tumour size greater than $30 \mathrm{~mm}$ often used as a cut-off to recommend primary medical therapy (Snelling et al., 2004).

Several studies were published concerning the accuracy of mammography, sonography, and clinical examination for breast tumor size measurement (Fornage et al., 1987; Finlayson et al., 2000; Hieken et al., 2001; Snelling et al., 2004; Parajuly et al., 2010). Overall, the accuracy of CBE is influenced by patient and observer factors and is not useful for clinically occult tumors. Mammographic assessment of tumour size may be less reliable than clinical or ultrasound measurement due to variation in the distance between the tumor and the film, indistinct tumor boundaries, compression during examination or because standard imaging projections do not capture the maximum tumor diameter (Hieken et al., 2001; Sofi et al., 2012). However, to date ultrasound measurement of tumour size is considered a more accurate imaging modality (Snelling et al., 2004).

The BreastLight performance for accurate masses size estimation in women requires validation. Our hypothesis was that, the measurement of lesion size using BreastLight apparatus could be useful and more accurate as adjuvant 
to the other modality systems. The purpose of our study was therefore to compare the performance of BreastLight with mammography, sonography and clinical breast examination at discriminating between a tumour greater or smaller than $40 \mathrm{~mm}$ was analysed.

\section{Materials and Methods}

Between July 2011 and September 2013, 500 women with mastalgia presented to the outpatient clinic of Mansoura University Hospital. The mean (SE) age at enrollment was $37 \pm 4.2$ years, with a range of 19 to 55 years.

Clinical, mammographic, and sonography assessments of the masses size were performed prior to BreastLight. All masses were measured clinically, mammographically, and sonographically by three different experienced physicians blinded to the results of other modalities. All of the masses were documented in two dimensions, and the maximum size was compared with the maximum BreastLight size. In women who did not have a detectable mass with a specific measurement method, the tumor size was assumed to be $0 \mathrm{~cm}$ for the diagnostic method concerned. In this series, of 500 women, mammography examinations were performed in 395 women older than 30 years and sonography was performed in 105 women younger than 30 years due to the breast density. The mammograms were reviewed with regard to breast density by a single expert radiologist.

A written informed consent obtained from all the participants. Furthermore, this study was approved by the ethics committee of Medical Faculty, Shahid Sadoughi University of Medical Sciences and Health Services, Yazd, Iran.

\section{Clinical breast examination}

Examination of the breast was done by the same surgeon in all cases. With the patient in the supine position and one arm raised, the physician thoroughly palpates breast tissue on the raised-arm side in the superficial, intermediate, and deep tissue planes; axilla, supraclavicular area, neck, and chest wall at least for 5 minutes. In premenopausal women, the CBE is best performed the week following menses, when breast tissue is least engorged. The larger diameter was used as the clinical masses size.

\section{Mammography}

Conventional film-screen mammography was performed with at least two views per breast (mediolateral oblique and craniocaudal views). Additional views or spot compression views were obtained where appropriate. The largest dimension was determined from these views with a ruler. Mammograms were obtained with dedicated mammography units (Alpha RT Imaging, General Electric Medical Systems, Milwaukee). Patients younger than 30 years were excluded, because mammography is not performed in this age group.

\section{Sonography}

High-resolution ultrasound was performed by an experienced physician. All breast sonographic evaluations were performed with the patient in a supine position for the medial parts of the breast and in a contralateral posterior oblique position with arms raised for the lateral parts of the breast. The sonograms were obtained using real-time technique with 7.5 to $13 \mathrm{MHz}$ transducers (Siemens Elegra, GE Logic 500, and ATL HDI 5000; Siemens, Erlangen, Germany). The probe was held orthogonal to the skin and the two largest diameters of the mass were measured with the machine's electronic calipers and recorded on the static images (results were rounded up to the nearest millimeter). For measurement purposes, the mass edge was defined as the end of the hypoechoic mass before the wide border denoting the transition between the mass and the healthy surrounding tissue.

\section{Statistical analysis}

The Statistical Package for the Social Sciences (SPSS) version 12.0 (SPSS, Inc., Chicago, IL, USA) was used for all statistical analyses. Differences between the masses size as assessed with the different methods (mammography, sonography, and clinical examination) and the BreastLight detection were analyzed using $\mathrm{X}^{2}$-trend test with $\mathrm{p}<0.05$ considering to be statistically significant.

\section{Results}

A total of 500 women were included in the study. The participants' mean age was 39.2 years (standard deviation [S.D.] 13.1 years). In Table 1 are shown that, most (more than 50\%) of the larger lesions than $1 \mathrm{~cm}$ assessed in CBE were identified on Breastlight. Our study shows that the performance of the Breast light in detection lesions smaller than or equal to $1 \mathrm{~cm}$ assessed by CBE is $4.4 \%$, for masses $1-2 \mathrm{~cm}, 2-3 \mathrm{~cm}, 3-4$, and $>4 \mathrm{~cm}$ are $52.2 \%, 59.3 \%, 63.2 \%$ and $65 \%$, respectively. The performance of Breast light in detection masses assessed by CBE significantly increased with larger masses $(\mathrm{p}=0.0001)$. Therefore, these results demonstrated a statistically significant positive correlation between Breastlight and CBE.

In Table 2 are shown that, most (more than 50\%) of the larger lesions than $1 \mathrm{~cm}$ assessed in Mammography were identified on Breastlight. Our study shows that the performance of the Breastlight in detection lesions smaller than or equal to $1 \mathrm{~cm}$ assessed by Mammography is $7.7 \% \%$, for masses $1-2 \mathrm{~cm}, 2-3 \mathrm{~cm}, 3-4$, and $>4 \mathrm{~cm}$

Table 1. Performance of the BreastLight in Detection of Masses Assessed by CBE

\begin{tabular}{|c|c|c|c|c|c|c|}
\hline \multirow[t]{2}{*}{ Detection } & \multicolumn{6}{|c|}{ Lesions Size } \\
\hline & $<1$ & 2 & 3 & 4 & $>4$ & Total \\
\hline $\begin{array}{l}\text { Lesions size by } \mathrm{CBE} \\
\text { Breast light }\end{array}$ & 207 (52.4) & $59(14.9)$ & $91(23.1)$ & $28 \quad(7.1)$ & $(2.5)$ & $395(100)$ \\
\hline Detected & $16(7.7)$ & $26(44.1)$ & $71(78.1)$ & $24(85.7)$ & $10(100.0)$ & $147 \quad(37.2)$ \\
\hline Not detected & $191(92.3)$ & $33(55.9)$ & $20(21.9)$ & $4(14.3)$ & $\begin{array}{ll}0 & (0.0)\end{array}$ & $248 \quad(62.8)$ \\
\hline
\end{tabular}


Table 2. Performance of the BreastLight in Detection of Masses Assessed by Mammography

\begin{tabular}{|c|c|c|c|c|c|c|}
\hline \multirow[t]{2}{*}{ Detection } & \multicolumn{6}{|c|}{ Lesions Size } \\
\hline & $<1$ & 2 & 3 & 4 & $>4$ & Total \\
\hline $\begin{array}{l}\text { Lesion size by Mammography } \\
\text { Breast light }\end{array}$ & 207 (52.4) & $59(14.9)$ & $91(23.1)$ & $28 \quad(7.1)$ & $10 \quad(2.5)$ & $395(100)$ \\
\hline Detected & $16(7.7)$ & $26(44.1)$ & $71(78.1)$ & $24(85.7)$ & $10(100.0)$ & $147 \quad(37.2)$ \\
\hline Not detected & $191(92.3)$ & $33(55.9)$ & $20(21.9)$ & $4(14.3)$ & $\begin{array}{ll}0 & (0.0)\end{array}$ & $248 \quad(62.8)$ \\
\hline \multicolumn{7}{|c|}{ Table 3. Performance of Breastlight in Detection of Masses Assessed by Sonography } \\
\hline \multirow[t]{2}{*}{ Detection } & \multicolumn{6}{|c|}{ Lesions Size } \\
\hline & $<1$ & 2 & 3 & 4 & $>4$ & Total \\
\hline $\begin{array}{l}\text { Lesions size by Sonography } \\
\text { BreastLight }\end{array}$ & $32(30.5)$ & $9(8.6)$ & $35(33.3)$ & $22(21.0)$ & $7(16.7)$ & $105(100)$ \\
\hline Detected & $4(12.5)$ & $2(22.2)$ & $11(31.4)$ & $10(45.5)$ & $4(57.1)$ & $31(29.5)$ \\
\hline Not detected & $28(87.5)$ & $7(77.8)$ & $24(68.6)$ & $12(54.4)$ & $3(42.9)$ & $74 \quad(70.5)$ \\
\hline
\end{tabular}

are $44.1 \%, 78.1 \%, 85.7 \%$ and $100 \%$, respectively. The performance of Breast light in detection masses assessed by Mammography significantly increased with larger masses $(\mathrm{p}=0.0001)$. Therefore, these results demonstrated a statistically significant positive correlation between Breastlight and Mammography in detection breast masses.

In Table 3 are shown that, most (more than 50\%) of the larger lesions than $1 \mathrm{~cm}$ assessed in Sonography were identified on Breastlight. Our study shows that the performance of the Breast light in detection lesions smaller than or equal to $1 \mathrm{~cm}$ assessed by Sonography is $12.5 \%$, for masses $1-2 \mathrm{~cm}, 2-3 \mathrm{~cm}, 3-4$, and $>4 \mathrm{~cm}$ are $22.2 \%, 31.4 \%, 45.5 \%$ and $57.1 \%$, respectively. Overall, BreastLight detected $29.5 \%$ of masses detected by sonography. Therefore, these results demonstrated, there are not statistically significant positive correlation between BreastLight and Sonography.

\section{Discussion}

Tumor size is commonly measured by palpation, but this method varies among observers, is influenced by many factors such as skin thickness, depth of mass, edema, and obesity and is prone to overestimation of tumor size (Shoma et al., 2006).

To date, only a few investigators have assessed the association between masses size and detection rate for different modality systems. It reported that the size of breast masses influence radiologist and surgeon sensitivity, it may also influence the tumor detection rate of different modality systems. Furthermore, Breast cancer survival and treatment is strongly associated with masses size at detection. It is reported that clinical assessment must take into account skin thickness and the depth of the tumor within the breast. In addition, clinicians can only palpate two (length and width) axes of a three-dimensional tumor, compared to ultrasound, which can provide threedimensional measurement including assessment of tumor depth (Snelling et al., 2004). Also, studies have shown that clinicians tend to overestimate the size of breast tumors (Pain et al., 1992; Finlayson et al., 2000; and Snelling et al., 2004). Previous studies results suggest that the tumor detection rate may be associated with lesion size (Malich et al., 2003). Highest detection rates were observed for 1030-mm tumor masses and for invasive ductal carcinomas and noninvasive cancers (Malich et al., 2003). Ultrasound is a well-established method to assessment of masses and tumor size at presentation. It is considered a more accurate measure of pathologic tumor size compared to clinical measurement. It is estimated that clinical palpation tends to overestimate tumor size and has the largest standard deviation of the difference, while ultrasound and mammography tend to underestimate masses and tumor size and have a similar standard deviation of the difference (10). Underestimation of tumor size may result in incomplete excisions, leading to re-excisions or higher local recurrence rates. Overestimation of tumor size may result in excisions that are larger than required, leading to a poor cosmetic outcome (Jiang et al., 2007).

In this study we found masses size influence BreastLight performance. Its highest detection rates have observed for $>4$-mm masses. It found that Breastlight performance is similar with mammography and clinical examination in determining breast masses size. Also, it found that BreastLight cannot to detect masses that identified by sonography. However, it shown that sonography had underestimation in masses size assessment.

In 56 of the patients the size of the lesions was estimated by the use of callipers and the detection rates using the breast illumination method were $100 \%$ for tumors with $>2 \mathrm{~cm}$ and $83.3 \%$ for tumors with $<2 \mathrm{~cm}$ (Bundred et al., 1986). Recently, Labib et al reported that the BreastLight had sensitivity, specificity, positive predictive value, negative predictive value and total accuracy of $93.0 \%, 73.7 \%, 91.4 \%, 77.8 \%$ and $88.2 \%$, respectively in detection of breast cancer (Labib et al., 2013). Published trials on Breastlight demonstrate that light-based technology can deliver high sensitivity levels $(82 \%)$ in lesions over $1.5 \mathrm{~cm}$ and useful levels $(29 \%)$ in non palpable lesions (Iwuchukwu et al., 2009). In a study counducted in the sundeland city hospital with 115 breasts; of these 84 (73\%) were Breastlight negative and 31 (27\%) were BreastLight positive. Breastlight positive lumps were significantly larger than Breastlight negative lumps ( $\mathrm{p}=0.02$,); Breastlight positive lumps were on average $18 \mathrm{~mm}$ compared with Breastlight negative lumps which were $11 \mathrm{~mm}$ on average (Iwuchukwu et al., 2009). In the subgroup of 18 malignant lumps, there was no significant difference between the size of detected (median $26.5 \mathrm{~mm}$ ) and not detected masses by Breastlight (median $23.5 \mathrm{~mm}$ ) 
Seyed Mostafa Shiryazdi et al

$(\mathrm{P}=0.9)$. Breastlight detection rate for malignant lesions was between $0.7 \mathrm{~cm}$ and $3.6 \mathrm{~cm}$ (Iwuchukwu et al., 2009).

The percutaneous methods including laser, radiofrequency, and cryoablation of eradicating breast tumors rely on accurate sonographic guidance for their efficiency. However, It has been observed that the sonographic underestimation of the histological tumour size (Hieken et al., 2001; Bosch et al., 2003; and Shoma et al., 2006). With regard to breastlight, our study concurs with previous authors, an underestimation of the sonography result of $>4 \mathrm{~mm}$, although this was not found to be significant (Table 3). Also, the mammographic size estimation is also negatively affected by breast density. The study by Hieken et al. (2001) have shown a size underestimation with mammography, which was attributed to the high compression of the breast during the examination. It is estimated that the tumour size with mammography and sonography was underestimated in $14 \%$ and $18 \%$ of the results respectively (Boetes et al., 1995).

Ib conclusion, breastLight can detect breast masses that can be identified by mammography or clinical examination. BreastLight examination is a noninvasive and accurate way to evaluate primary masses size with high patient acceptance. However, BreastLight assessment should be used as an accurate adjunct to clinical examination in outpatient breast clinics.

\section{References}

Azizun-Nisa, Bhurgri Y, Raza F, Kayani N (2008). Comparison of ER, PR and HER-2/neu (C-erb B 2) reactivity pattern with histologic grade, tumor size and lymph node status in breast cancer. Asian Pac J Cancer Prev, 9, 553-6.

Boetes C, Mus RD, Holland R, et al (1995). Breast tumors: comparative accuracy of MR imaging relative to mammography and US for demonstrating extent. Radiology, 197, 743-7.

Bosch AM, Kessels AG, Beets GL, et al (2003). Preoperative estimation of the pathological breast tumour size by physical examination, mammography and ultrasound: a prospective study on 105 invasive tumours. Eur J Radiol, 48, 285-92.

Bundred N, Levack P, Watmough DJ, Watmough JA (1986). Preliminary results using computerised tele-Diaphanography for the investigation of breast disease. Br J Hospital Med, 37, 70-1.

Iwuchukwu O, Dordea M, Keaney N (2009).A clinical investigation to develop an evidence base for the use of Breastlight in examining the breast. Sunderland City Hospital. http://acare.cz/img/soubory/breastlight2.pdf.

Fornage BD, Toubas O, Morel M (1987). Clinical, mammographic, and sonographic determination of preoperative breast cancer size. Cancer, 60, 765-71.

Fouladi N, Pourfarzi F, Mazaheri E, et al (2013). Beliefs and behaviors of breast cancer screening in women referring to health care centers in northwest Iran according to the champion health belief model scale. Asian Pac J Cancer Prev, 14, 6857-62.

Finlayson CA, MacDermott TA(2000). Ultrasound can estimate the pathologic size of infiltrating ductal carcinoma. Arch Surg, 135, 158-9.

Hieken TJ, Harrison J, Herreros J, Velasco JM (2001). Correlating sonography, mammography, and pathology in the assessment of breast cancer size. Am J Surg, 182, 351-4.
Pain JA, Ebbs SR, Hern RP, Lowe S, Bradbeer JW (1992). Assessment of breast cancer size: a comparison of methods. Eur J Surg Oncol, 18, 44-8.

Parajuly SS, Lan PY, Yan L, Gang YZ, Lin L (2010). Breast elastography: a hospital-based preliminary study in China. Asian Pac J Cancer Prev, 11, 809-14.

Shoma A, Moutamed A, Ameen M, Abdelwahab A (2006). Ultrasound for accurate measurement of invasive breast cancer tumor size. Breast J, 12, 252-6.

Snelling JD, Abdullah N, Brown G, et al (2004). Measurement of tumour size in case selection for breast cancer therapy by clinical assessment and ultrasound. Eur J Surg Oncol, 30, 5-9.

Sofi GN, Sofi JN, Nadeem R, et al (2012). Estrogen receptor and progesterone receptor status in breast cancer in relation to age, histological grade, size of lesion and lymph node involvement. Asian Pac J Cancer Prev, 13, 5047-52.

Tham TM, Iyengar KR, Taib NA, Yip CH (2009). Fine needle aspiration biopsy, core needle biopsy or excision biopsy to diagnose breast cancer-which is theideal method? Asian Pac J Cancer Prev, 10, 155-8.

Jiang YX, Liu H, Liu JB, et al (2007). Breast tumor size assessment: comparison of conventional ultrasound and contrast-enhanced ultrasound. Ultrasound Med Biol, 33, 1873-81.

Labib NA, Ghobashi MM, Moneer MM, Helal MH, Abdalgaleel SA (2013). Evaluation of breastlight as a tool for early detection of breast lesions among females attending National cancerinstitute, cairo university. Asian Pac J Cancer Prev, 14, 4647-50.

Luparia A, Mariscotti G, Durando M, et al (2013). Accuracy of tumour size assessment in the preoperative staging of breast cancer: comparison of digital mammography, tomosynthesis, ultrasound and MRI. Radiol Med, 118, 1119-36.

Malich A, Sauner D, Marx C, et al (2003). Influence of breast lesion size and histologic findings on tumor detection rate of a computer-aided detection system. Radiology, 228, 851-6.

Nguansangiam S, Jesdapatarakul S, Tangjitgamol S (2009). Accuracy of fine needle aspiration cytology from breast masses in Thailand. Asian Pac J Cancer Prev, 10, 623-6. 